\title{
Trends in the Incidence of Biopsy-Proven Glomerular Diseases in the Adult Population in Central Poland in the Years 1990-2010
}

\author{
Ilona Kurnatowska ${ }^{a}$ Dorota Jędrzejka ${ }^{a} \quad$ Aneta Małyska ${ }^{a} \quad$ Małgorzata Wągrowska-Danilewicz $^{b}$ \\ Marian Danilewicz $^{b}$ Michał Nowicki ${ }^{a}$ \\ a Department of Nephrology, Hypertension and Kidney Transplantation and ${ }^{\mathrm{b}}$ Division of Nephropathology, \\ Department of Pathology, Medical University of Łódź, Poland
}

Key Words

Epidemiology $\cdot$ Renal biopsy $\cdot$ Glomerulonephritis

\begin{abstract}
Background: There is a paucity of epidemiological data on biopsy-proven glomerulonephritis (GN) in Poland. The aim of this study was to assess the epidemiology of renal diseases based on histological diagnosis in the region of Central Poland over the last two decades. Methods: Retrospective analysis of the results of 746 consecutive native kidney biopsies performed in the Caucasian adults from 1990 to 2010 in a single tertiary nephrology center serving an area of Central Poland. Results: Primary GN was found in $81.4 \%$ of all biopsies. The mesangioproliferative GN including IgA nephropathy was the most frequent type of primary GN (51.2\%). Membranoproliferative GN was diagnosed between 1990 and 2000 more frequently than in the following decade (26.7 vs. $7.3 \%, \mathrm{p}<0.001)$. There was a significant increase in the incidence of FSGS (4.8 vs. 17.3\%, p < 0.001) and MCD (5.1 vs. $11.2 \%, p<0.001$ ) over time. Secondary GN was documented in $18.6 \%$ of biopsies and lupus nephritis was the most frequent cause (34.5\%). Conclusion: Our analysis showed the decrease in the incidence of membranoproliferative GN with the parallel increase of FSGS and MCD over the last twenty years. Mesangioproliferative GN including IgA nephropathy remains the most frequent type of $\mathrm{GN}$ observed in our region.

Copyright $\odot 2012$ S. Karger AG, Basel
\end{abstract}

\section{Introduction}

The incidence of glomerular diseases (GD) varies according to the population, demographic characteristics, environmental factors, socioeconomic status, prevalence of infectious diseases and historical time period $[1,2]$. Knowledge of the epidemiology of glomerulopathies among persons living in a particular region may provide an important background for clinical practice by offering directions to both treatment and prognosis. Current epidemiological data of kidney diseases from some European countries [3-7] and from outside Europe [8] are available mostly from renal biopsy registries. The analysis of these data show a changing pattern of adult glomerulopathies over the years.

The information about the incidence and distribution pattern of different forms of glomerulonephritis (GN) in Poland has been scarce. The Łódź region is geographically distinct region of Central Poland with stable, homogenous, almost entirely Caucasian, population of $\sim 2.6$ million.

The aim of this study was to report on long-term trends in epidemiology of GD in adult Polish population, based on histopathological diagnosis, in the region of Central Poland over the period of the last 20 years (19902010).

\section{KARGER}

Fax +41613061234 E-Mail karger@karger.ch www.karger.com

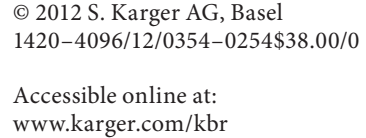

Prof. Michał Nowicki, MD

Department of Nephrology, Hypertension and Kidney Transplantation

Medical University of Łódź

Kopcińskiego 22, PL-91-156 Łódź (Poland)

Tel. +48 42677 6709, E-Mail nefro@wp.pl 
Table 1. Prevalence of different forms of primary and secondary glomerulonephropathies in the years 1990-2010

\begin{tabular}{|c|c|c|c|c|}
\hline & $\begin{array}{l}\text { Total } \\
\mathrm{n}(\%)\end{array}$ & $\begin{array}{l}\text { 1990-2000 } \\
\mathrm{n}(\%)\end{array}$ & $\begin{array}{l}\text { 2001-2010 } \\
\mathrm{n}(\%)\end{array}$ & $\mathrm{p}$ value \\
\hline Primary glomerular diseases & 607 (81.4) & 375 (61.7) & $232(38.3)$ & \\
\hline Mesangioproliferative glomerulonephritis (with IgAN) & $311(51.2)$ & $193(51.4)^{*}$ & $118(50.9)$ & n.s. \\
\hline Membranoproliferative glomerulonephritis & $117(19.3)$ & $100(26.7)$ & $17(7.3)$ & $<0.001$ \\
\hline Membranous glomerulonephritis & $68(11.2)$ & $40(10.6)$ & $28(12.0)$ & n.s. \\
\hline Focal segmental glomerulosclerosis & $58(9.5)$ & $18(4.8)$ & $40(17.3)$ & $<0.001$ \\
\hline Minimal change diseases & $45(7.4)$ & $19(5.1)$ & $26(11.2)$ & $<0.01$ \\
\hline Crescentic glomerulonephritis & $6(0.9)$ & $4(1.0)$ & $2(0.8)$ & n.s. \\
\hline Poststreptococcal acute glomerulonephritis & $2(3.3)$ & $1(2.7)$ & $1(4.3)$ & n.s. \\
\hline Secondary glomerular diseases & $139(18.6)$ & $61(13.9)$ & $78(25.2)$ & \\
\hline Lupus nephritis & $48(34.5)$ & $22(36.0)$ & $26(33.3)$ & n.s. \\
\hline Necrosing vasculitides & $30(21.6)$ & $15(24.5)$ & $15(19.2)$ & n.s. \\
\hline Secondary amyloidosis & $34(24.4)$ & $17(27.8)$ & $17(21.8)$ & n.s. \\
\hline Diabetic nephropathy & $13(9.4)$ & $1(1.6)$ & $12(15.4)$ & n.s. \\
\hline $\begin{array}{l}\text { Others (Schönlein-Henoch disease, light-chain deposit } \\
\text { disease, Alport syndrome, Goodpasture’s syndrome, } \\
\text { antiphospholipid syndrome, Fabry disease) }\end{array}$ & $14(10.1)$ & $6(9.8)$ & $8(10.6)$ & n.s. \\
\hline
\end{tabular}

* Including 16 IgAN cases (17.6\% of all 91 MesGN cases) diagnosed in the years 1990-1995 when immunofluorescence was not performed routinely that might have led to underdiagnosis of IgAN and 37 IgAN (36.3\% among total $102 \mathrm{MesGN})$ diagnosed in the years 1996 and 2000 when IF was routinely performed.

\section{Material and Methods}

The records of renal biopsies performed in Caucasian patients $>18$ years old at a single tertiary university nephrology center in Central Poland from January 1990 till December 2010 were retrospectively analyzed. The following data for each patient were recorded: age, sex and histological diagnosis. Kidney biopsy in the center was carried out in subjects with proteinuria with or without micro- or macrohematuria, hematuria with arterial hypertension or rapidly progressive renal insufficiency. Ultrasound needle guidance and the biopsy gun were used for biopsies.

Unique for this study was that over the whole study period, the same two consultant pathologists with special interest in renal pathology were interpreting all kidney biopsy specimens. All biopsy specimens were analyzed by light microscopy. Immunofluorescence (IF) was available for more than $70 \%$ of specimens being less frequently performed in the first of the analyzed decades, since 1995 the tissues simples were routinely stained for IgG, IgM and IgA, C3, C1q, light chains kappa and lambda. Electron microscopy was not routinely performed and therefore only $50 \%$ of renal biopsy specimens were analyzed with this method, most in the second decade. The histological findings were categorized for the purpose of this analysis as primary GN (PGN) and secondary GN (SGN). PGN was further classified into seven pathologies, i.e. minimal change disease (MCD), mesangioproliferative nephropathy (MesGN) including IgA nephropathy (IgAN), focal segmental glomerulosclerosis (FSGS), membranous nephropathy (MN), membranoproliferative GN (MPGN), crescentic glomerulonephritis (CGN) and poststreptococcal GN. Diabetic nephropathy, lupus nephritis (LN), vasculitis, GN caused by dysgammaglobu- linemia or paraproteinemia such as renal amyloidosis, light-chain deposit disease including AL amyloidosis, monoclonal immunoglobulin deposition disease and light chain cast nephropathy and less common nephropathies as: Schönlein-Henoch disease, Alport syndrome, Goodpasture's syndrome, antiphospholipid syndrome, Fabry disease were classified as secondary glomerulopathies. The data were divided into two inclusive 10-year time frames, i.e. 1990-1999 and 2000-2010. The patients were divided into three groups according to age into young age group, i.e. 18-30 years of age, middle age (31-65 years old) and elderly that included patients $>65$ years old.

\section{Results}

During the period of 20 years (1990-2010) 746 native kidney biopsies were performed. There were 439 biopsies performed in the 1990s and 307 between 2000 and 2010. The mean age of the patients was $40.5 \pm 20.8$ years (335 F; $411 \mathrm{M})$. Most of kidney biopsies were performed in patients between 30 and 65 years of age (519 biopsies 69.6\%), 188 between 18 and 30, and 39 above 65 years of age. PGN was found in $81.4 \%$ of all biopsies and secondary in $18.6 \%$ with an approximate annual incidence of all GN in the area of 14.3 per million inhabitants. The distribution of major histological types of GD in our popu- 


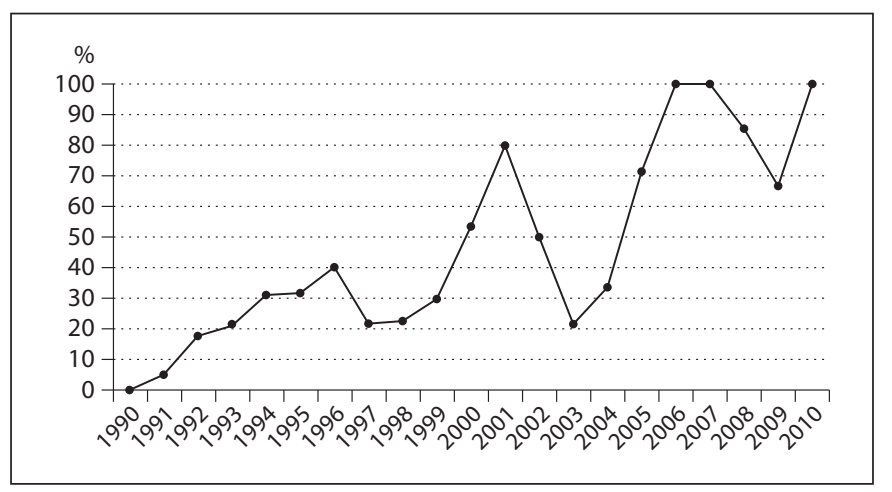

Fig. 1. Ratio of IgA nephropathy to all mesangioproliferative nephropathies in the years 1990-2010.

lation is shown in table 1 . MesGN including IgA nephropathy were the most frequently encountered GD over the whole period of 20 years, but the proportion of IgAN among the MesGN increased systematically. IgAN was rarely diagnosed in the early nineties, but since 1995, when the IF has been routinely performed, the frequency of IgAN among MesGN increased and was approximately between $30-40 \%$ of MesGN in 1995 and 2000, and reached $100 \%$ in the end of the second decade (fig. 1). The MPGN was the second most common condition over the study period, but its prevalence significantly decreased in the latter 10 years, from $26.7 \%$ between 1990 and 2000 to $7.3 \%$ from 2000 to $2010, \mathrm{p}<0.001$, (fig. $2 \mathrm{a}$ ). The increase of FSGS (17.3 vs. $4.8 \%, \mathrm{p}<0.001$; fig. $2 \mathrm{~b})$ and MCD (11.2 vs. $5.1 \%, p<0.01$; fig. 2 c) was noticed in $2000-2010$ in comparison to previous decade. The frequency of $\mathrm{MN}$ was $11.2 \%$ of PGN and did not change significantly during the whole analyzed period. It was the most frequent PGN in patients over 65 years of age. The CGN was diagnosed in 6 patients and poststreptococcal acute GN was observed in only 2 patients during the study.

Lupus nephritis was the most frequent SGN (34.5\%) and was diagnosed mainly among young females. In $58.1 \%$ of cases it was found in patients below 30 years old. Vasculitis was diagnosed more frequently in the older age group, in $28.1 \%$ of all SGN between 30 and 65 years old and in 30\% over 65 years. Secondary amyloidosis was the most frequently found in patients over 65 years old (60\% of all SGN in this age). Diabetic kidney disease was diagnosed in only 13 cases, the Schönlein-Henoch disease in 6 patients, lightchain deposit disease in 3 patients, Alport syndrome in 2 patients, and there was one case of Goodpasture's syndrome, antiphospholipid syndrome and Fabry disease.
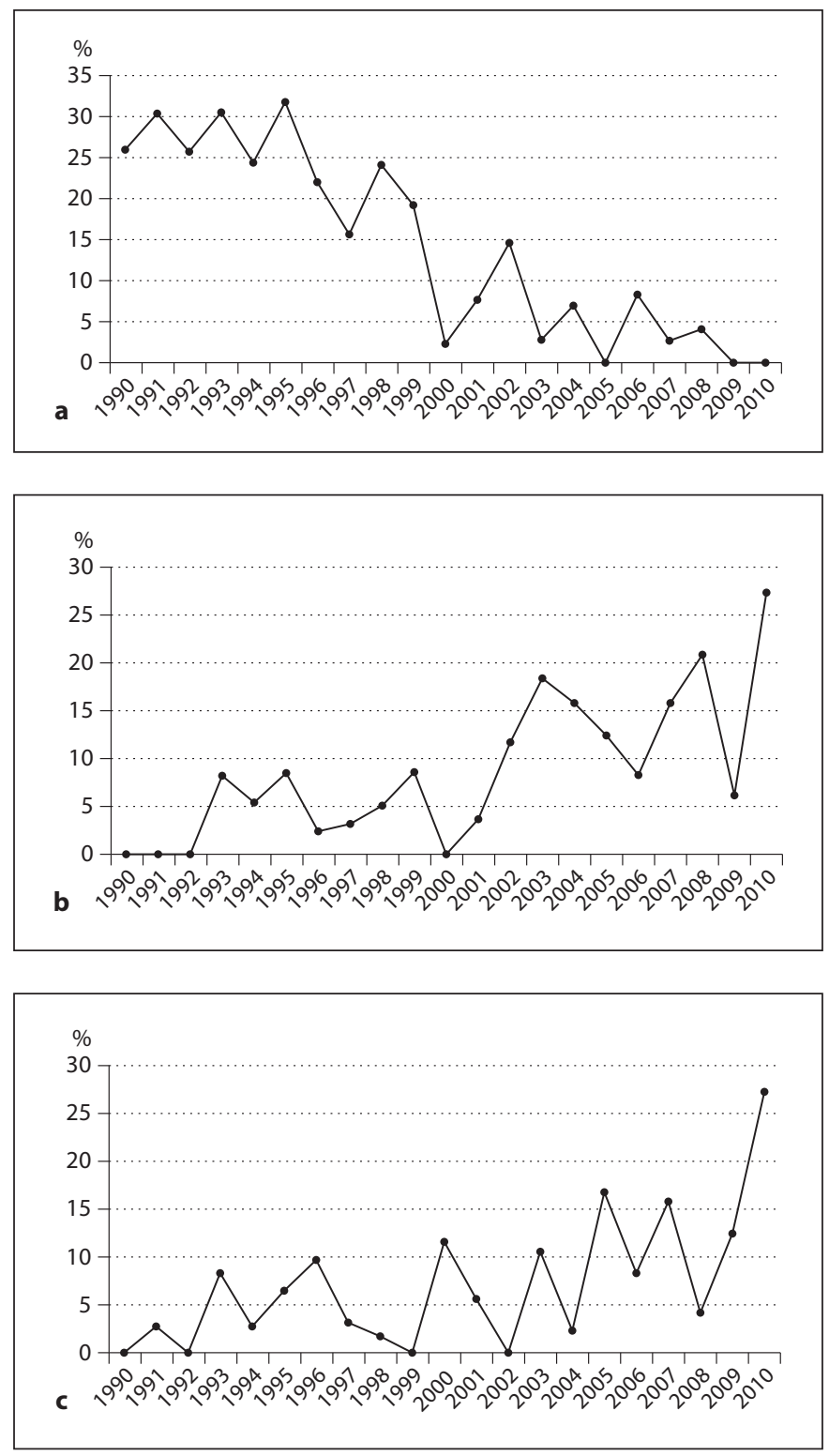

Fig. 2. Ratio of different types of glomerulopathy to all primary glomerulonephropathies in the years 1990-2010. a Membranoproliferative glomerulopathy. b Focal segmental glomerulosclerosis. c Minimal change disease.

\section{Discussion}

Our analysis provides the information about the trends in demographics and prevalence of biopsy-proven glomerulopathies during the period of the 20 last years in Central Poland. In most European countries, similar to our region, MesGN including IgAN has been the most common over the twenty years studied $[3,5]$. Further- 
more its incidence has not changed over that period. In our study, mesangial non-IgA nephropathy was diagnosed very often in the last decade of the twentieth century. This phenomenon may be partly due to less frequent use of immunofluorescence in the first (mainly between 1990 and 1995) than in the second decade. The number of cases of IgAN diagnosed in biopsy specimens increased systematically together with an increase of IF performing and in the last decade reaching $100 \%$ of MesGN diagnoses. IgAN is the most common glomerular disease in our region, similar to other European countries, with the prevalence ranging from 30 to $60 \%$ [9-11] and peak incidence in young and middle aged people.

MPGN was the second most frequent PGN over the studied period and was found in almost one quarter of all biopsies in our population. Its incidence, however, systematically decreased and was not recognized in the last 2 years. A similar tendency in other European countries was previously reported $[4,9]$. Since the incidence of this type of GD is clearly infection-related such a trend could be attributed to improved standard of living, better organization of health system, decrease in hepatic viral infection and early introduction of antibiotics in the treatment of common infections.

$\mathrm{MN}$ was the third most popular diagnosis of GD in our population and was the most frequent among patients older than 65 . Its incidence did not change significantly over those 20 years. A similar and constant frequency of MN was reported by Rychlík et al. [3] in the Czech Republic that is in the similar population. Interestingly, in the analysis of Hanko et al. [7] in the Irish population over a 30 -year period, the proportion of PGN due to $\mathrm{MN}$ fell significantly. The currently available literature is inconsistent in reporting on incidence of $\mathrm{MN}$ as some authors reported no significant change [12], some an increase in diagnoses [13], and others observed a decrease [14].

The incidence of FSGS and MCD during the last twenty years in our population was similar to other European countries [3, 4], but was higher than in Northern Ireland [7]. Interestingly, the prevalence of FSGS and MCD significantly increased in the second decade. This phenomenon has not been previously reported in Europe, but found in the USA $[1,10,12,15]$. It has been well documented that the incidence of FSGS is higher in the African American population [1]. On the other hand, more recent data suggested that the incidence of this type of GD within Caucasian populations in the USA is also increasing $[12,14]$. The proportion of FSGS increased from 2.9 to $20 \%$ in the Caucasian population in Minnesota from 1974 to 2003 [12] and in our entirely Caucasian population from $4.8 \%$ of all primary glomerular diseases in $1990-2000$ to $17.3 \%$ in $2001-2010$. The reasons for the differing patterns in renal biopsy epidemiology are not completely clear. It seems probable that there is a substantial difference in the differentiation of FSGS from other primary glomerular diseases, particularly from MCD. Additionally, the increase in MCD diagnoses in our adult population was observed at the same time and it is well known that a proportion of MCD may be in fact undiagnosed FSGS. Various factors can explain more frequent recognition of FSGS such as genetic predisposition, lower birth weight, obesity or infections, e.g. human immunodeficiency virus (HIV) [15].

Our data confirm the observations of other authors that PGN was diagnosed most frequently among males, whereas SGN was most common among women mainly due to more frequent incidence of lupus nephritis $[3,6]$. LN was generally the most common secondary glomerulopathy affecting mostly young women. In contrast, vasculitis was more common among men, particularly in those over 30 years of age and secondary amyloidosis was most frequent finding in patients over 65 years. The incidence of these diseases did not change significantly over whole study period, however, was diagnosed slightly more often than in Czechs [3].

Diabetic nephropathy was an infrequent finding in our study that was clearly the result of an unavoidable selection bias caused by the reluctance of nephrologists to carry out biopsies in patients with long-lasting diabetes and presence of other signs (e.g. diabetic retinopathy) suggesting of diabetic nephropathy.

Our study reveals a similar incidence pattern of major glomerulopathies as in other European countries. It is rather difficult to provide definitive epidemiological data on the frequency of various forms of GN for several reasons: first, the renal biopsy indication policy varies from one center to another, second, renal biopsy is often not performed when the likelihood of its therapeutic implications is low (intermittent hematuria, non-nephrotic proteinuria, shrunken kidneys, advanced kidney function impairment).

Our study has several limitations. First of all it is retrospective and the data are based on histopathology. A part of the histopathological diagnosis is based on light microscopy only, without IF (mainly in 1990-1995) or electron microscopy. We were also not able to analyze clinical aspects like the indications for kidney biopsy, complications after the procedure or clinical outcome. We cannot give the precise diagnosis of some SGN, main- 
ly associated with vasculitis, due to lack of serological markers in some cases. Also the calculation of cases of GNC per million population (PMP values) is difficult to calculate because the study was not nationwide but regional and therefore a catchment area could not be precisely defined.

In conclusion, our analysis of kidney biopsy findings gives the first epidemiological information on the prevalence of different glomerulopathies in an adult Caucasian population in Central Poland over a long observation pe- riod of 20 years. The MesGN including IgAN remains the major chronic PGN. A decrease in the incidence of membranoproliferative GN with a parallel increase of FSGS and MCD was observed in our population. Lupus nephritis was the most common secondary glomerulopathy. The changing pattern of glomerular diseases in our region during the last twenty years prompts the implementation of a national biopsy registry in Poland and other countries of the region.

\section{References}

1 Korbet M, Genchi R, Borok Z, Schwartz MM: The racial prevalence of glomerular lesions in nephritic adults. Am J Kidney Dis 1996;27:647-651.

2 Halevy D, Radhakrishnan J, Appel GB: Racial and socioeconomic factors in glomerular disease. Semin Nephrol 2001;21:403-410.

\3 Rychlík I, Jančová E, Tesař V, Kolský A, Lácha J, Stejskal J, Stejskalová A, Dušek J, Herout V: The Czech registry of renal biopsies: occurrence of renal diseases in the years 1994-2000. Nephrol Dial Transplant 2004; 19:3040-3049.

$\checkmark 4$ Covic A, Schiller A, Volovat C, Gluhovschi G, Gusbeth-Tatomir P, Petrica L, Caruntu ID, Bozdog G, Velciov S, Trandafirescu V, Bob F, Gluhovschi C: Epidemiology of renal disease in Romania: a 10 year review of two regional renal biopsy databases. Nephrol Dial Transplant 2006;21:419-424.

5 Wirta O, Mustonen J, Helin H, Pasternack A: Incidence of biopsy-proven glomerulonephritis. Nephrol Dial Transplant 2008;23: 193-200
6 Naumovic R, Pavlovic S, Stojkovic D, BastaJovanovic G, Nesic V: Renal biopsy registry from a single centre in Serbia: 20 years of experience. Nephrol Dial Transplant 2009;24: 877-885.

7 Hanko JB, Mullan RN, O’Rourke DM, McNamee PT, Maxwell AP, Courtney AE: The changing pattern of adult primary glomerular disease. Nephrol Dial Transplant 2009; 24:3050-3054.

8 McGrogan1 A, Franssen CFM, de Vries CS: The incidence of primary glomerulonephritis worldwide: a systematic review of the lit erature. Nephrol Dial Transplant 2011;26: 414-430.

$\checkmark 9$ Simon P, Ramee MP, Boulahrouz R, Stanescu C, Charasse C, Ang KS, Leonetti F, Cam G, Laruelle E, Autuly V, Rioux N: Epidemiologic data of primary glomerular diseases in western France. Kidney Int 2004;66:905908.

10 Rivera F, López-Gómez JM, Pérez-García R: Spanish Registry of Glomerulonephritis: clinicopathologic correlations of renal pathology in Spain. Kidney Int 2004;66:898904.
11 Gesualdo L, Di Palma AM, Morrone LF, Strippoli GF, Schena FP, Italian Immunopathology Group, Italian Society of Nephrology: The Italian experience of the national registry of renal biopsies. Kidney Int 2004; 66:890-894.

12 Swaminathan S, Leung N, Lager DJ, Melton LJ 3rd, Bergstralh EJ, Rohlinger A, Fervenza FC: Changing incidence of glomerular disease in Olmsted County, Minnesota: a 30year renal biopsy study. Clin J Am Soc Nephrol 2006;1:483-487.

13 Riviera F, Lopez-Gomez JM, Perez-Garcia R: Frequency of renal pathology in Spain 19941999. Nephrol Dial Transplant 2002;17: 1594-1602.

14 Braden GL, Mulhern JG, O’Shea MH, Nash SV, Ucci AA Jr, Germain MJ: Changing incidence of glomerular diseases in adults. Am J Kidney Dis 2000;35:878-883.

15 Dragovic D, Rosenstock JL, Wahl SJ, Panagopoulos G, DeVita MV, Michelis MF: Increasing incidence of focal segmental glomerulosclerosis and an examination of demographic patterns. Clin Nephrol 2005;63: $1-7$. 